\title{
A Study on Large Bubble Motion and Liquid Film in Vertical Pipes and Inclined Narrow Channels
}

\author{
F. Behafarid ${ }^{1}$, K. E. Jansen ${ }^{2}$ and M. Z. Podowski ${ }^{1}$ \\ ${ }^{1}$ Rensselaer Polytechnic Institute, Troy, New York, USA. \\ ${ }^{2}$ University of Colorado, Boulder, Colorado, USA.
}

\begin{abstract}
The objective of this paper is to discuss the results of a combined physical and computational analysis of the dynamics of large deformable bubbles in conduits of different geometries and orientations. The situations analyzed included a vertical circular pipe and a narrow rectangular channel of different inclination angles. Threedimensional simulations were performed at a DNS-scale using the PHASTA code combined with the level set method for interface tracking. The results of computer simulations for Taylor bubble flow in a vertical pipe have been verified against a simplified theoretical model and validated against available general evidence deduced from various experimental studies. The predictions for bubbles flowing along inclined rectangular channels have been validated against the experiments of Maneri [1970]. Several modeling and numerical issues have been investigated, including the effect of a liquid microfilm between the bubble and the wall above it, and the impact of the blending region arising from the level-set model formulation on the accuracy of results.
\end{abstract}

Keywords: Two-Phase Flow, Level Set Method, DNS, Liquid Microfilm, Taylor Bubbles, Narrow Channels.

\section{INTRODUCTION}

Experimental and numerical studies of bubbles moving in conduits of different orientations and shapes have many practical applications. The development of methods to accurately predict the bubble shape and motion is important for a wide range of systems used by the gas/oil industry, nuclear power plants, chemical processing plants, microchannels employed by pharmaceutical devices, etc.

The purpose of this paper is to combine theoretical analysis with advanced computer simulation methods, to perform a comprehensive study on the mechanisms governing the motion of large deformable bubbles in narrow vertical and inclined conduits.

The computer simulations were performed using a complete three-dimensional first-principle model of gas/liquid flow. The DNS-scale model has been implemented in the PHASTA (Parallel Hierarchic Adaptive Stabilized Transient Analysis) computer code, with a modified Level-Set Method (LSM) used to capture the liquid/gas interface. Whereas previous twophase flow applications of PHASTA have been mainly for dispersed bubbly flows, the current study has been focused on the analysis of the fluid mechanics of large bubbles confined between, and interacting with, solid walls. The results of the combined theoretical investigations and computer simulations have been extensively tested parametrically, verified against other models and validated against experimental data.

\section{BACKGROUND}

A thorough literature review has been performed to identify the existing experimental evidence about bubbles moving in narrow channels of various orientations, and to identify a meaningful frame of reference for the validation of the proposed model. The needed information included: detailed geometry and dimensions, fluid properties, and flow conditions. Another issue of practical importance was concerned with the compatibility of the experimental time scale with the size of the corresponding computational problem, to assure that meaningful numerical simulations could be performed at reasonable computational costs. A brief overview of selected relevant information is given below.

Local flow measurements of vertical upward bubbly flow in an annulus have been reported by Hibiki et al [2003], including the following parameters: void fraction, interfacial area concentration and interfacial velocity, liquid velocity and turbulence intensity.

Yadigaroglu et al. [2008] published a set of experimental data for bubbly plumes and jets. Some of these data were ensemble- or phase-averaged to filter out large-scale meandering and oscillations of coherent structures and to make the time-dependent small-scale effects and local stress terms more visible.

Wilmarth et al. [1994] studied adiabatic concurrent vertical and horizontal two-phase flows of air and water through narrow rectangular channels. Their study was mainly focused on flow regime transition criteria. 
Fourar et al. [1995] experimented with air/water flows in narrow horizontal channels simulating material fractures. Air and water were injected through alternating capillary tubes to produce a uniform distribution at the inlet.

Barnea [1990] studied the shape of Taylor bubbles and compared different analytical models. One of the conclusions drawn from this work was that predicting the curved geometry of bubble's nose is essential for the overall modeling consistency.

Polonsky et all [1999] experimentally studied the motion of Taylor bubble in vertical pipes in stagnant, upward and downward liquid flows. They also measured the distribution of liquid velocities in front of the bubble and inside the liquid film.

Finally, a series of experiments was reported by Maneri and Zuber [1974] on the measurements of the velocity of rising bubbles in narrow channels of various inclination angles. Since this study provided details of the experimental setups and documented the results of measurements for a wide range of bubble rise velocities, bubble sizes and inclination angles, selected experimental data sets have been used as a frame of reference for the validation of the current model(s). The main emphasis in model validation has been on the effect of inclination angle on bubble shape and velocity. For specified inclination angles, two sets of numerical simulations have been performed for each case using different approaches to the modeling of the liquid film between the bubble and the walls.

Numerical simulations were performed using 8129 processors at the RPI's CCI Blue Gene supercomputer based on a finite element method and unstructured, boundary-layer meshing. The computations continued until a steady state was reached. The computational cost for each simulation (one bubble size at one inclination angle) was around 100000 CPU-hours.

\section{OVERVIEW OF COMPUTATIONAL METHODOLOGY}

\subsection{PHASTA Code}

PHASTA is a parallel, hierarchic (between 2ndand 5th orders of accuracy, depending on function choice), adaptive, stabilized (finite element) transient analysis DNS flow solver for both incompressible and compressible flows [Whiting et al 2001, Jansen et al 2000]. PHASTA has been applied to turbulent flows ranging from validation benchmarks to complex flows. Interestingly, the computational options available in PHASTA also include advanced anisotropic adaptive algorithms [Sahni, et al 2006] and LES/DES models [Tejada-Martinez et al 2005].

The two-phase version of PHASTA utilizes the Level-Set method to define the interface between the gas and liquid phases [Adalsteinsson et al., 1999]. The combined DNS/Level-Set model has been extensively tested and validated for various two-phase flow problems and conditions, including a study on bubble dynamics [Nagrath et al., 2005] and the effect of turbulence on bubble shape and distribution [Bolotnov et al., 2011a]. PHASTA DNS/LSM capabilities were also used recently to perform two-phase flow simulations aimed at studying the evolution of the flow of gas released through a breach in the fuel rod cladding into the liquid sodium coolant in a complex geometry of narrow channels of a Gen. IV nuclear reactor ([Behafarid et al., 2010], [Bolotnov et al., 2010], [Bolotnov et al., 2011b], [Behafarid, 2012], [Behafarid et al., 2012], [Behafarid et al., 2013]).

\subsection{Governing Equations}

The numerical solution algorithm implemented in PHASTA is based on the finite element formulation. Details of the spatial and temporal discretizations of the incompressible Navier-Stokes equations in PHASTA have been described by Whiting [1999] and Nagrath [2004]. Following the stabilized formulation of Taylor et al. [1998], the residuals of the continuity and momentum equations have been combined together. The stabilization and its parameters were described by Franca and Frey [1992] and by Whiting and Jansen [2001]. The overall stabilized finite element formulation has been described in detail by Rodriguez [2009] and Jansen et al. [1999]. The surface tension force has been computed as a local interfacial force density used in the Brackbill's CST model [Brackbill et al., 1992]. In this method, a continuous body force is applied in the direction normal to the interface (only inside a very small region around it, i.e. the blending region) with a magnitude based on the surface tension coefficient and the local curvature of the interface.

\subsection{Formulation of Level Set Model}

The "level set method", introduced by Sussman ([Sussman et al., 1998], [Sussman et al., 1999a], [Sussman et al., 1999b], [Sethian 1999]) models the interface as the zero level set of a smooth function, $\varphi$, representing the signed distance from the interface. The scalar $\varphi$ is convected by a moving fluid according to

$\frac{D \varphi}{D t}=\frac{\partial \varphi}{\partial t}+\vec{u} \cdot \nabla \varphi=0$

where $u$ is the fluid velocity. Typically, the liquid phase is indicated by a positive level set and gas phase by a negative level set.

Since using a step function to represent the jump in gas/liquid physical properties leads to poor numerical results, a smoothed Heaviside kernel function, $H_{\varepsilon}$, has been used to smoothly blend the properties in a region in vicinity of the interface (known as blending region) 


$$
H_{\varepsilon}(\varphi)=\left\{\begin{array}{cc}
0, & \varphi<-\varepsilon \\
\frac{1}{2}\left[1+\frac{\varphi}{\varepsilon}+\frac{1}{\pi} \sin \left(\frac{\pi \varphi}{\varepsilon}\right)\right], & |\varphi|<\varepsilon \\
1, & \varphi>\varepsilon
\end{array}\right.
$$

where $\varepsilon$ is half of the blending region's thickness. Density, $\rho$, and viscosity, $\mu$, are defined as

$$
\begin{aligned}
& \rho(\varphi)=\rho_{l} H_{\varepsilon}(\varphi)+\rho_{g}\left(1-H_{\varepsilon}(\varphi)\right) \\
& \mu(\varphi)=\mu_{l} H_{\varepsilon}(\varphi)+\mu_{g}\left(1-H_{\varepsilon}(\varphi)\right)
\end{aligned}
$$

Subscripts $l$ and $g$ represent liquid and gas; respectively. Since the varying fluid velocities throughout the flow field distort the level set contours, the distance field may not be correct throughout the domain, especially in the regions far from the interface. Therefore, a re-distancing operation corrects the level set contours by solving the following PDE

$$
\frac{\partial d}{\partial T}=S(\varphi)[1-|\nabla d|]
$$

where $d$ represents the corrected distance field and $T$ is the pseudo time used to obtain an asymptotic steadystate solution. Eq.(5) can also be rewritten as

$$
\frac{\partial d}{\partial T}+\vec{\omega} \cdot \nabla d=S(\varphi)
$$

where $\omega$ is the pseudo-velocity

$$
\vec{\omega}=S(\varphi) \frac{\nabla d}{|\nabla d|}
$$

And $S(\varphi)$ is defined as

$$
S(\varphi)=\left\{\begin{array}{cc}
{\left[\frac{\varphi}{\varepsilon}+\frac{1}{\pi} \sin \left(\frac{\pi \varphi}{\varepsilon}\right)\right],} & |\varphi|<\varepsilon \\
1, & \varphi>\varepsilon
\end{array}\right.
$$

The original level set field, $\varphi$, is used as the initial condition for the second scalar, $d$, which is then convected at the pseudo-velocity, $\vec{\omega}$.

The procedure described above restores the distance field to $\nabla d= \pm 1$, but it does not alter the location of the interface $(\varphi=0)$ since the pseudo-velocity, $\vec{\omega}$, is zero at the interface. Then, $\varphi$, is updated using the steady state solution of $d$. It is important to notice that in the re-distancing phase, the information always travels from the interface to the boundaries of the computational domain. Therefore, the level set method does not require the specification of any particular boundary conditions.

To improve the mass conservation, an additional constraint is applied during the re-distancing to prevent the interface from moving ([Sussman et al., 1999a], [Sussman et al., 1999b]). This constraint basically preserves the volume of each phase during the re- distance step. In each cell, the volume of each phase is

$$
V_{i j}^{k}=\int_{\Omega^{e}} H_{\varepsilon}\left(d^{k}\right) d x
$$

where $d^{k}$ is the value of the distance field, at the $k_{\mathrm{th}}$ iteration. Small changes in the zero level set (interface location) between pseudo time steps of $T^{0}$ and $T^{k}$ can be approximated by

$$
\begin{aligned}
V_{i j}^{k}-V_{i j}^{0} & \approx\left(T^{k}-T^{0}\right) \int_{\Omega^{e}} \frac{\partial H_{\varepsilon}\left(d^{0}\right)}{\partial T} d x \\
& \approx \int_{\Omega^{e}} H_{\varepsilon}^{\prime}\left(d^{0}\right)\left(d^{k}-d^{0}\right) d x
\end{aligned}
$$

where

$H_{\varepsilon}^{\prime}(\varphi)=\frac{\partial H_{\varepsilon}(d)}{\partial d}=\left\{\begin{array}{cc}0, & |d|>\varepsilon \\ \frac{1}{2 \varepsilon}\left[1+\cos \left(\frac{\pi d}{\varepsilon}\right)\right], & |d| \leq \varepsilon\end{array}\right.$

Finally, to minimize the volume change, each new $d_{i j}^{k}$ should satisfy the following condition

$\int_{\Omega^{e}} H_{\varepsilon}^{\prime}\left(d^{0}\right)\left(d^{k}-d^{0}\right) d x=0$

The value of the current level set, $\tilde{d}_{i j}^{k}$, is projected onto the new level set field, $d_{i j}^{k}$, as

$$
d_{i j}^{k}=\tilde{d}_{i j}^{k}+\lambda_{i j}\left(T^{k}-T^{0}\right) H_{\varepsilon}^{\prime}\left(d^{0}\right)
$$

The scaling function $\lambda_{i j}$, which is assumed to be constant within each cell, is computed by

$$
\lambda_{i j}=\frac{-\int_{\Omega^{e}} H_{\varepsilon}^{\prime}\left(d^{0}\right)\left(\frac{\tilde{d}^{k}-d^{0}}{T^{k}-T^{0}}\right) d x}{-\int_{\Omega^{e}}\left(H_{\varepsilon}^{\prime}\left(d^{0}\right)\right)^{2} d x}
$$

Note that when convergence of the distance field is achieved, the $\tilde{d}_{i j}^{k}$ and $d_{i j}^{k}$ are the same and $\lambda_{i j}$ is zero.

The consistency and accuracy of the current levelset algorithm implemented in the PHASTA code were extensively tested before for a variety of cases [Nagrath, 2004; Rodriguez, 2009, 2013; Bolotnov et al., 2011; Behafarid et al., 2013]. It has been shown that the present method is capable of preserving the conservation of gas volume with a required level of accuracy. Interestingly, a similar conclusion can also be drawn from a study in which a similar methodology was implemented in another computer code [Wierzbicki et al., 2006]. Needless to say, additional testing and verification have been performed for the current problem. 


\section{THREE-DIMENSIONAL PREDICTIONS OF THE MOTION OF A LONG BUBBLE RISING IN A VERTICAL PIPE}

\subsection{Results of 3-D Model Predictions}

A complete three-dimensional (3-D) model has been formulated to simulate the flow of a Taylor bubble in a $2 \mathrm{~m}$ long vertical pipe, $2.5 \mathrm{~cm}$ in diameter, filled with stagnant water at $20{ }^{\circ} \mathrm{C}$. A boundary layer mesh was generated to discretize the computational domain totaling 27 million elements with an average element size of $0.25 \mathrm{~mm}$ and 100 elements across the diameter of the pipe. The first layer of the boundarylayer mesh was $0.1 \mathrm{~mm}$ in height. At initial step of calculations, an elongated bubble was prescribed, as shown in Figure 1.

A snapshot of the time-dependent PHASTA simulation results is shown in Figure 2. As can be seen, the flow and the interface are smooth at the bubble front while at the bottom the flow becomes turbulent, making the interface unstable and leading to the formation of small bubbles behind in the wake of the Taylor bubble. The approximately constant bubble rise velocity, evaluated based on the location of the bubble front, was $16.44 \mathrm{~cm} / \mathrm{s}$.

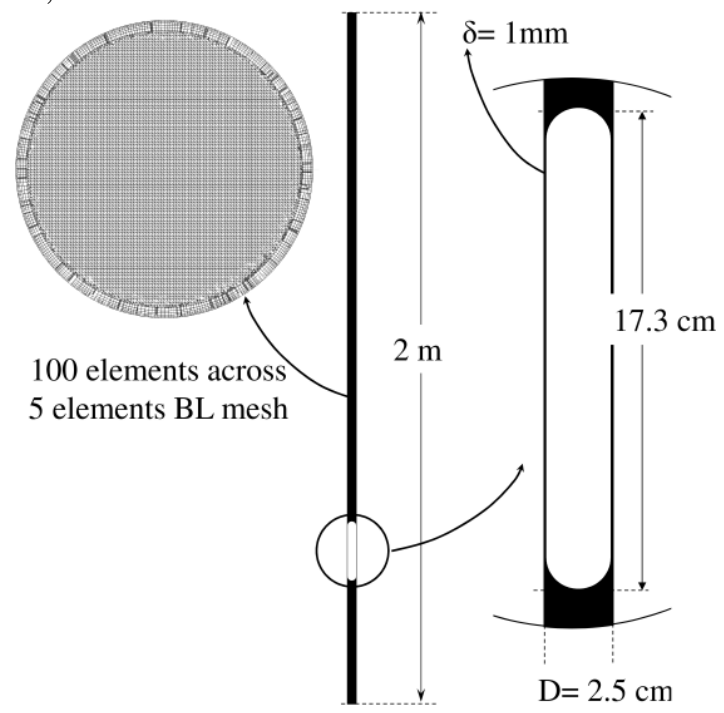

Figure 1. Computational domain, mesh and initial phase indicator. White region indicates the gas phase.

Figure 3 shows the velocity profiles across the pipe at different locations indicated as $a, b$ and $c$ in Figure 2.

The gas/liquid interface locations, corresponding to the zero values of the level-set function, are shown by short vertical lines on both sides of the Taylor bubble.

All three locations experience some common features. For example, due to the downward liquid flow along most of the bubble length and a negligible shear induced by the gas phase, the liquid film velocity is always negative and its derivative is always insignificant at the interface.

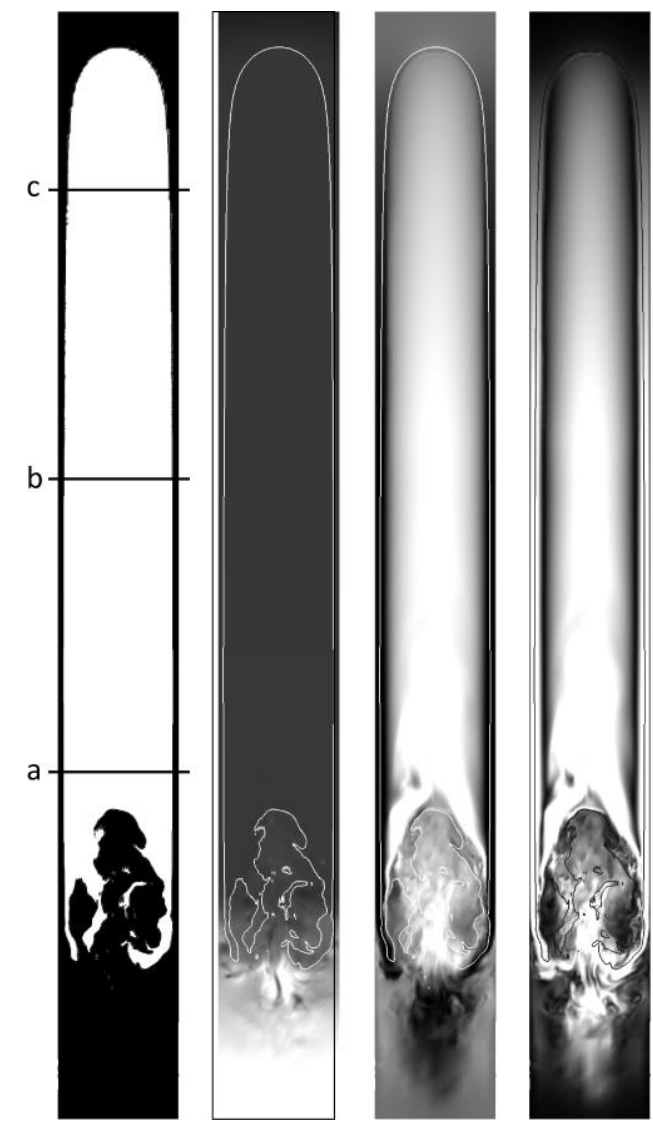

Figure 2. Snapshots of the phase indicators, pressure, velocity magnitude and axial velocity (from left to right) at $t=0.175 \mathrm{~s}$.

The liquid film is laminar at all three locations, but the gas flow tends to become turbulent at the bottom of the bubble.

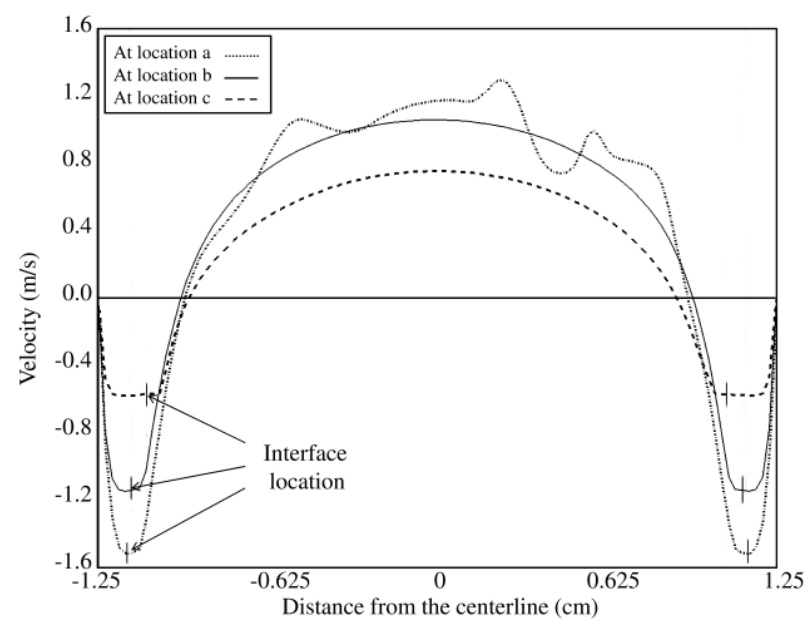

Figure 3. A snapshot of the velocity profiles across the domain (at locations a, b and c, shown in Fig 2).

Figure 4 shows a snapshot of the velocity distribution at different locations along the bubble. It is interesting to notice that the gas flow field can also be 
considered laminar at the top-half of the bubble.

Figure 5 presents the phase indicator at nine time steps. It is obvious that very soon after the buoyancy kicks in and pushes the bubble up, the rising velocity reaches a steady value.
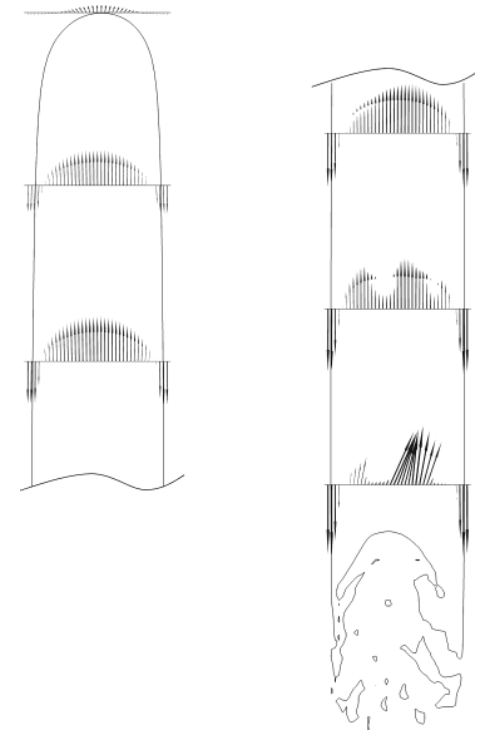

Figure 4. Snapshot of the velocity profile across the domain at different locations.

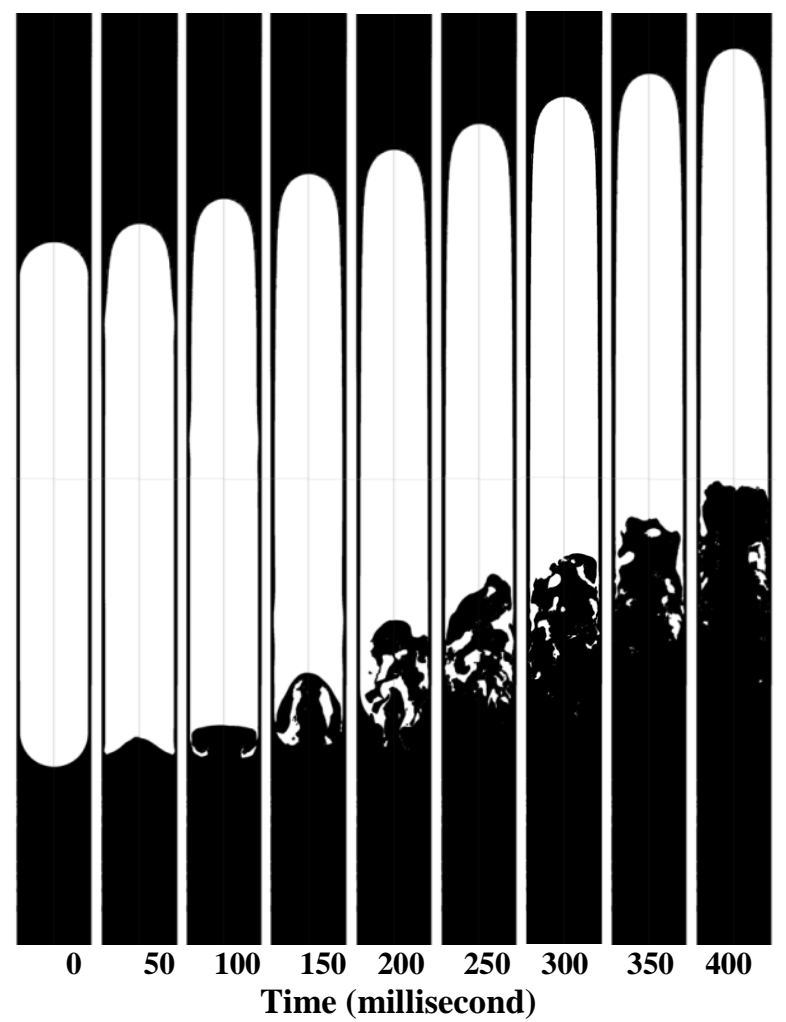

Figure 5. Snapshots at regular time intervals of the predicted locations of a Taylor bubble rising in a pipe.
An interesting issue arising from the examination of the results shown in Figure 5 is concerned with the impact of small dispersed bubbles on the predicted Taylor bubble shape and velocity. Since to fully resolve the shape and distribution of such bubbles a computational mesh with 15 to 30 elements across each small bubble would be required, the computational cost of such simulations would be tremendous. Furthermore, since in reality the break-up process is very sensitive to the actual experimental conditions, statistical averaging would still be required to draw meaningful conclusions about the bubbles' shape and motion.

On the other hand, it has been shown in the process of the current study as well as in earlier works [Rodriguez et al., 2009], that the impact of small bubbles on the shape and motion of elongated gas/liquid interfaces is relatively small. In the case of long Taylor bubbles, it has already been firmly demonstrated ([Bendiksen, 1985], [Barnea, 1990], [Anglart and Podowski, 2002]) that the main factors governing the shape and velocity of such bubbles are the curvature around the bubble tip and the force balance between buoyancy and shear along the liquid film. Thus, the effect of small dispersed bubbles and of the fluctuating interface at the bubble's tail play minor roles, at least as long as the Taylor bubble is sufficiently long. In particular, both experimental observations and the results of simulations using computer models such as the current one, clearly show that the fluctuations do not destabilize either the bubble shape or the rising velocity. Thus, an important lesson learned from this study is that the main factor to assure good predictive capabilities of computational models is their ability to properly capture the geometry of the liquid film along Taylor bubbles all the way between the tip of the bubble and the formation of its unstable tail.

\subsection{Analysis and Model Verification/Validation}

The purpose of the results shown in Section 4.1 was to demonstrate the physical and numerical consistency of the combined DNS/Level-Set model implemented in the PHASTA code, prior to its application to a more complex problem of large bubble flow in inclined rectangular conduits. Whereas the physical conditions used in the analysis were not related to a particular experiment, however it was deemed important to compare the PHASTA simulations against both general available data for the flow of bubbles in vertical tubes and theoretical results based on simplified modeling assumptions. Another important factor is an assessment of the sensitivity of predictions to selected features of the level-set numerical method. 
(A) Bubble Rise Velocity based on Phenomenological Models

It is commonly understood that the equilibrium velocity of long bubbles rising in a pipes filled with a stagnant liquid mainly depends on the: pipe diameter, liquid viscosity, liquid and gas densities, and surface tension. In particular, the bubble rise velocity, $\bar{u}_{g}$, can be expressed by a well-known general relationship [Zukoski 1966],

$\bar{u}_{g}=C_{\infty}\left(N_{f}, E o, \theta\right) \sqrt{g^{\prime} D}$

where $N_{f}$ is the dimensionless inverse viscosity and $E \ddot{O}$ is the dimensionless inverse surface tension (or Eötvös Number), respectively given by

$$
\begin{aligned}
& N_{f}=\frac{D^{\frac{3}{2}} g^{\prime \frac{1}{2}} \rho_{l}}{\mu_{l}} \\
& E \ddot{o}=\frac{\rho_{l} g^{\prime} D^{2}}{\sigma}
\end{aligned}
$$

where, $\rho_{l}$ and $\mu_{l}$ are liquid density and liquid viscosity; respectively, $\sigma$ is the surface tension, $D$ is the pipe diameter and,

$$
g^{\prime}=\frac{g \Delta \rho}{\rho_{l}}
$$

Zukoski reported that long bubbles rising in vertical tubes are normally stable to external perturbations. Nicklin et al. [1962] observed that front shape of a long bubble is similar to a prolate spheroid, and is nearly independent of the bubble length. On the other hand, the film region is clearly affected by the length of the bubble and is controlled by the balance between friction, buoyancy and curvature of the bubble front. The bubble rear shape depends on the viscous force and the surface tension effects. Based on a simplified Bendiksen [1985] correlation (typographically corrected by Fabre et al [1992]), for large values of $N_{f}$ the coefficient, $C_{\infty}$, in Eq.(15) can be expressed as

$$
C_{\infty}=0.344 \frac{1-0.96 e^{(-0.0165 E \ddot{o})}}{\left[1-0.52 e^{(-0.0165 E \ddot{\theta})}\right]^{1.5}} \sqrt{1+\frac{20}{E \ddot{o}}\left(1-\frac{6.8}{E \ddot{o}}\right)}
$$

Applying the expressions given by Eqs.(15)-(19) to the conditions used in the simulations discussed in Section 4.1, yields the results summarized in Table 1.

As can be seen, the bubble rise velocity of 16.88 $\mathrm{cm} / \mathrm{s}$ obtained from Eq.(15) agrees well with the PHASTA-predicted value of $16.44 \mathrm{~cm} / \mathrm{s}$, and the difference between the two results is less than $3 \%$.
Table 1. Parameters for the conditions used in the PHASTA simulations reported in Section 4.1.

\begin{tabular}{lll}
\hline Symbol & Definition & Value \\
\hline$g^{\prime}$ & Modified gravitational acceleration & 9.798 \\
$E \ddot{o}$ & Eotvos number & 84.25 \\
$N_{f}$ & Dimensionless inverse viscosity & 14147 \\
$C_{\infty}$ & Velocity Coefficient & 0.341 \\
$\bar{u}_{g}$ & Bubble rising velocity $(\mathrm{cm} / \mathrm{s})$ & 16.88 \\
\hline
\end{tabular}

(B) Average Liquid Film Thickness based on $\underline{\text { Phenomenological Models }}$

Extensive experimental studies have been carried out before on the measurements of liquid films between Taylor bubbles and tube walls. Due to technical difficulties associated with capturing details of the position-dependent liquid film thickness, the reported experimental data are typically based on a constant liquid film thickness averaged over the lower part of the length of the bubble. For instance, Karapantsios et al. [1995] proposed the following correlation valid for $9000<N_{f}<44000$

$$
\frac{2 \delta}{D}=0.428\left[\frac{\mathrm{Re}_{b}^{0.538}}{N_{f}^{2 / 3}}\right]
$$

where, $\delta$ is the liquid film thickness and $\operatorname{Re}_{b}$ is defined as

$$
\operatorname{Re}_{b}=\frac{\rho_{l} V_{b} D}{\mu_{l}} \approx 4\left(\frac{\rho_{l} \bar{V}_{l} \delta}{\mu_{l}}\right)
$$

Using the PHASTA-predicted bubble rise velocity of $16.44 \mathrm{~cm} / \mathrm{s}$, the Reynolds number given by Eq.(21) becomes, $\operatorname{Re}_{b} \approx 4700$, and the corresponding film thickness is, $\delta=0.87 \mathrm{~mm}$.

Another study for liquid film thickness around Taylor bubbles [Lewellin et al, 2011] recommended that for $N_{f}$ greater than 10000 , the film thickness is independent of this parameter and becomes only a fixed fraction function of the pipe diameter

$\frac{\delta}{D} \approx 0.04$

For the pipe diameter of $2.5 \mathrm{~mm}$ used in Section 4.1, the approximate liquid film thickness obtained from Eq.(22) is, $\delta \approx 1 \mathrm{~mm}$.

Since, as shown In Figure 3, the film thickness between the locations $3 \mathrm{~cm}$ and $15 \mathrm{~cm}$ below the tip of the bubble changed by nearly a factor of two, the estimates given by either Eq.(20) or Eq.(22) provide only very crud approximations, but are not capable of capturing the actual axial evolution of the liquid film 
thickness. It turns out, however, that a much more meaningful verification of the PHASTA-based computational model can be accomplished by using a theoretical result based on a simplified model of variable-thickness liquid film. Details are shown below.

\section{(C) Analytical Model of Local Liquid Film Thickness}

Several attempts have been made before to predict local aspects of the velocity field around Taylor bubbles. The purpose of most works in the past was to formulate complete stand-alone analytical approximations of flow around Taylor bubbles, to understand effects such as that of the shape of the bubble nose [Barnea, 1990] or of the the liquid velocity field in front of the bubble [Polonsky, 1999] on bubble velocity. The objective of the model presented in this section is to obtain an independent confirmation of the evolution of liquid film thickness along the Taylor bubble length predicted by the DNS PHASTA-based simulation, based on a purely analytical first-principle approach. To avoid using any approximated expressions for the flow around bubble nose, the model has been applied only to the thin film region, and the upstream liquid flow boundary conditions have been provided by a complete three-dimensional solution around the bubble tip.

Since in the present case, the liquid flow along the Taylor bubble is laminar and axisymmetric, its motion is governed by a two-dimensional momentum equation. Assuming that the film thickness is small compared to the pipe diameter, the momentum equation in the system of coordinates moving with the Taylor bubble can be written as

$\rho_{l}\left[\frac{\partial\left(u_{l}^{2}\right)}{\partial z}+\frac{\partial\left(u_{l} \cup_{l}\right)}{\partial y}\right]=-\frac{\partial p}{\partial z}+g \rho_{l}+\frac{\partial \tau}{\partial y}$

where $u_{l}(y, z)$ is the local liquid velocity, $p$ is pressure, $g$ is gravitational acceleration, $z$ is directed downwards, $y=0$ at the channel wall, and $y=\delta$ corresponds to the liquid/gas interface. Also, since the $z$ coordinate is along the liquid film flow direction, $\frac{\partial \tau}{\partial y} \leq 0$.

Since $\rho_{g}<<\rho_{l}$, the axial pressure gradient inside the bubble is negligible compared to the hydrostatic pressure gradient along the liquid film. Furthermore, the pressure distribution across the liquid film and gas region inside the Taylor bubble is nearly uniform (the effect of surface tension across the almost cylindrical gas/liquid interface along the bubble is very small [Anglart and Podowski, 2002(a), 2002(b)]. Thus, the net pressure gradient due to the combined hydrostatic and shear forces is very small compared to the hydrostatic term alone, and it can be ignored.
Consequently, Eq.(23) can be integrated across the film thickness, to obtain

$\int_{0}^{\delta} \frac{\partial\left(u_{l}^{2}\right)}{\partial z} d y+\int_{0}^{\delta} \frac{\partial\left(u_{l} \cup_{l}\right)}{\partial y} d y=g \delta+\frac{1}{\rho_{l}} \int_{0}^{\delta} \frac{\partial \tau}{\partial y} d y$

Then

$\left[\frac{d}{d z} \int_{0}^{\delta} u_{l}^{2} d y-\left[u_{l}(\delta)\right]^{2} \frac{d \delta}{d z}\right]+u_{l}(\delta) \mathrm{v}_{l}(\delta)=g \delta-\frac{\tau_{w}}{\rho_{l}}$

where $\tau_{w}>0$ is the magnitude of the wall shear stress.

Taking into account that

$\mathrm{v}_{l}(\delta)=\frac{d \delta}{d t}=\frac{d \delta}{d z} u_{l}(\delta)$

Eq.(25) can be simplified to

$\frac{d}{d z} \int_{0}^{\delta} u_{l}^{2} d y=g \delta-\frac{\tau_{w}}{\rho_{l}}$

Using a parabolic velocity profile of the axial liquid velocity profile is given by

$u_{l}=\left(u_{o}-u_{b}\right)\left[1-\left(1-\frac{y}{\delta}\right)^{2}\right]+u_{b}$

where $u_{l}(0, z)=u_{b}$. Other relationships are

$$
\begin{aligned}
& \bar{u}_{l}-u_{b}=\frac{2}{3}\left(u_{o}-u_{b}\right) \\
& \int_{0}^{\delta} u_{l}^{2} d y=\left[\frac{6}{5}\left(\bar{u}_{l}-u_{b}\right)^{2}+2\left(\bar{u}_{l}-u_{b}\right) u_{o}+u_{b}^{2}\right] \delta \\
& \frac{\tau_{w}}{\rho_{l}}=2 \frac{v\left(u_{o}-u_{b}\right)}{\delta}=3 \frac{v\left(\bar{u}_{l}-u_{b}\right)}{\delta}
\end{aligned}
$$

where $u_{o}$ is the liquid film velocity at the gas/liquid interface, and $\bar{u}_{l}$ is the axially-varying average liquid velocity inside the film.

Substituting Eqs. (28)-(31) into Eq.(27), we obtain the following differential relationship between the average liquid film velocity and thickness

$$
\begin{aligned}
\frac{6}{5} \frac{d}{d z}\{[ & {\left.\left[\left(\bar{u}_{l}-u_{b}\right)^{2}+\frac{5}{3}\left(\bar{u}_{l}-u_{b}\right) u_{o}+\frac{5}{6} u_{b}^{2}\right] \delta\right\}=} \\
& g \delta-3 v\left(\bar{u}_{l}-u_{b}\right) / \delta
\end{aligned}
$$

Since the liquid film average velocity can be related to the bubble velocity via the conservation of volume condition

$$
\bar{u}_{l}-u_{b}=\frac{(D-2 \delta)^{2}}{D^{2}-(D-2 \delta)^{2}} u_{b}=\frac{(D-2 \delta)^{2}}{4 \delta(D-\delta)} u_{b}
$$

the overall model can be converted into a single ordinary differential equation for the film thickness as a function of the axial distance along the liquid flow 


$$
\begin{gathered}
\frac{d}{d z}\left\{\left[\frac{(D-2 \delta)^{4}}{\delta^{2}(D-\delta)^{2}}+\frac{20}{3} \frac{(D-2 \delta)^{2}}{\delta(D-\delta)}+\frac{40}{3}\right] \delta\right\}= \\
\frac{40}{3} \frac{g}{u_{b}^{2}} \delta-10 \frac{\nu}{u_{b} \delta} \frac{(D-2 \delta)^{2}}{\delta(D-\delta)}
\end{gathered}
$$

The solution of Eq.(34) can be substituted into Eq.(33) and Eq.(29), to obtain the average and maximum velocities of the liquid film, respectively.

Eq.(34) can be readily integrated with a boundary condition given by a prescribed film thickness at any specific location along the bubble. However, since the model is applicable only under a "thin film" assumption, it cannot be used starting at the tip of the bubble. Furthermore, the accuracy of the calculated film thickness and velocity will improve if the starting point in the integration is moved further away from the nose of the bubble. This is shown in Figure 6, where the results of calculations by the simplified model are compared against the PHASTA-predicted distributions of the film thickness. As can be seen, whereas the agreement between the theoretical and computational results of is good in general, the difference becomes very small if the former model is limited to the lower section of the bubble length.

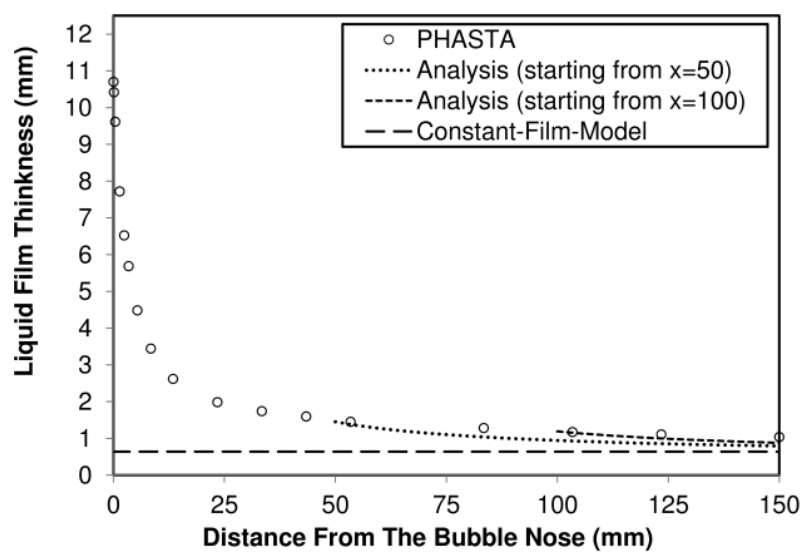

Figure 6. A comparison between the liquid film thickness predicted by PHASTA and the simplified theoretical model given by Eq.(34).

Interestingly, Eq.(34) can also be used to determine the asymptotic (uniform) film thickness which characterizes the conditions at the lower portion of very long Taylor bubbles. Namely, assuming that $\delta<<D / 2$ in Eq.(33), substituting the resultant expression into Eq.(32), and zeroing the LHS derivative, one obtains

$$
\delta=\sqrt[3]{\frac{3 v_{l} D u_{b}}{4 g}}
$$

For the bubble rise velocity, $u_{b}=16.44 \mathrm{~cm} / \mathrm{s}$, the film thickness obtained from Eq.(35) is, $\delta=0.65 \mathrm{~mm}$. A more accurate analysis based on using cylindrical coordinates predicts the asymptotic film thickness of $0.63 \mathrm{~mm}$. As can be seen the actual film thickness at a $15 \mathrm{~cm}$ distance from the tip of the bubble is about 1 . Thus, one concludes that the asymptotic expression given by Eq.(35) may considerably underestimate the liquid flim thickness even for relatively long bubbles.

(D) Effects of the LSM Blending Region on Numerical Prediction of the Liquid Film

The Level Set Method (LSM) interprets the interface as the zero level set of a smooth function representing the signed distance from the interface. One of the characteristic features of this method is that the properties of the two phases are smoothly averaged inside a region in the vicinity of the interface, which is called the blending region. Another important factor is that the surface tension force is computed as a local force applied inside the blending region.

The results presented in Figures 2 to 6 are based on blending region thickness, $\varepsilon=0.6 \mathrm{~mm}$. In order to study the effects of the blending region's thickness on the calculated liquid film thickness and the bubble rise velocity, another simulation was performed in which the blending region thickness was decreased from 0.6 $\mathrm{mm}$ to $0.3 \mathrm{~mm}$. The velocity profiles across the pipe at location-(b) in Figure 2, corresponding to both assumed thicknesses of the blending region, are shown in Figure 7 and Figure 8. In both figures, the blending regions are shown as gray areas.

By comparing the results shown in Figure 7 against those in Figure 8, it can readily noticed that the bubble rise velocity and the liquid film thickness are similar in both cases. Furthermore, the radial distributions across the pipe area, including both the gas and liquid regions are also nearly identical. Thus, one concludes that the blending region's thickness does not have a significant impact on the predictions. It can be explained by observing that the incompressible flow model accurately conserves the volume of both fluids, and small changes in the density distribution inside the liquid film region do not affect the downward volume flow rate or, consequently, the bubble rise velocity. 


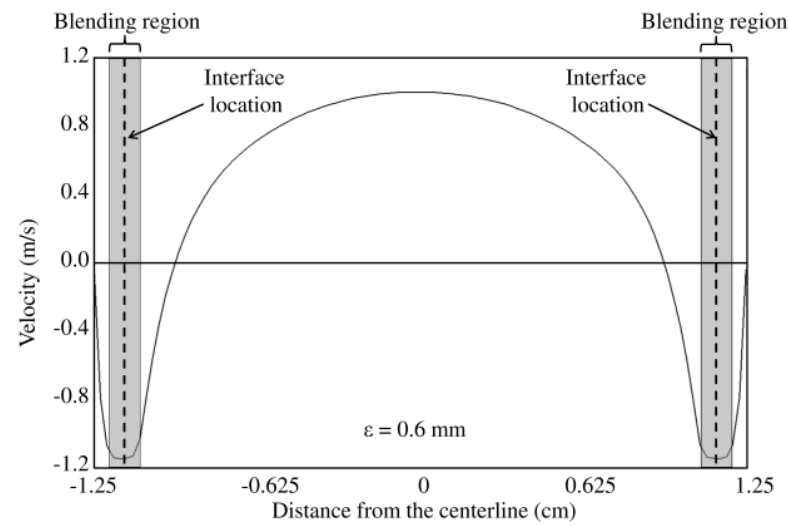

Figure 7. Velocity profile across the pipe at location(b) in Fig 2 for the blending region thickness of 0.6 $\mathrm{mm}$.

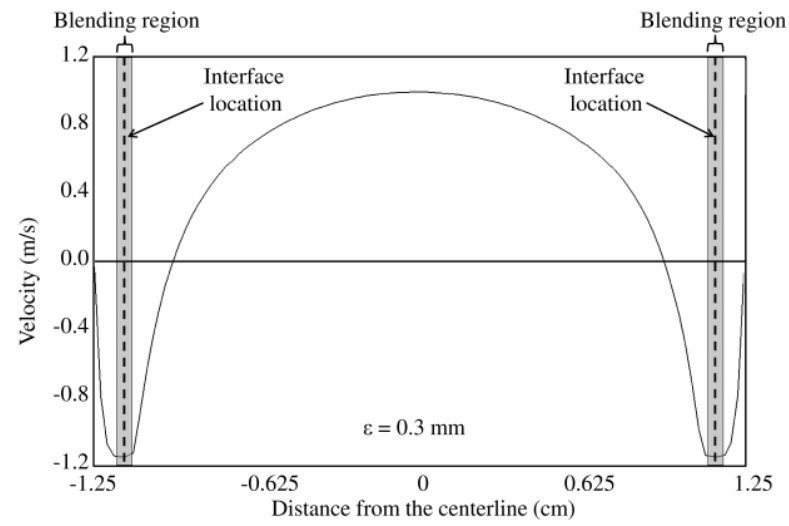

Figure 8. Velocity profile across the pipe at location(b) in Fig 2 for the blending region thickness of 0.3 mm.

\section{THREE-DIMENSIONAL MODELING OF LARGE BUBBLES RISING IN INCLINED NARROW CHANNELS}

\subsection{Problem Formulation and Original Results}

A very interesting and challenging two-phase flow problem, which is also of a considerable practical importance, deals with the modeling of flow of large deformable bubbles in conduits of various geometries and orientations. In particular, several theoretical and computational issues have to be resolved to achieve a good understanding of the fluid mechanics phenomena governing the motion of gas bubbles in narrow inclined channels, and to capture the effect of inclination angle, and of the thin liquid film between the bubble and the inclined wall above it, on bubble shape and velocity.

As it was mentioned in the Introduction section, the experimental conditions of Maneri [1970] (also, see Maneri and Zuber, 1974]) have been used as a reference in the formulation and validation of a 3-D model of the buoyancy-driven bubble motion in a quiescent liquid. A schematic of the experimental setup is shown in Figure 9. The available thorough documentation of the experimental setup includes details of conduit geometry, pressure, temperature, and boundary and initial conditions, thus allowing for the formulation of a complete physical and mathematical model. The proposed model has been subsequently implemented in the PHASTA code and numerically tested and validated.

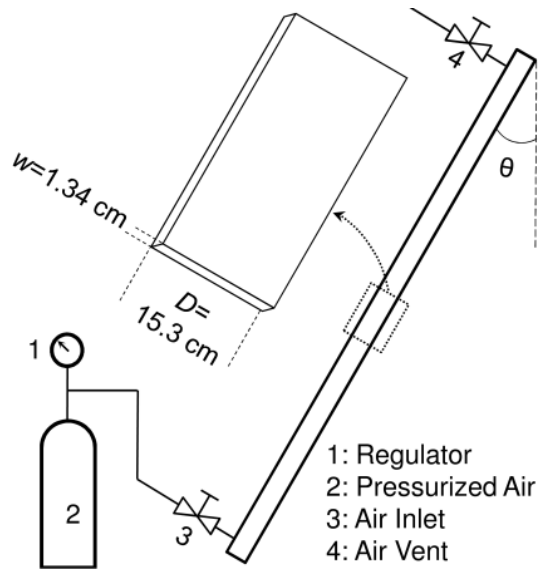

Figure 9. Experimental setup schematic [Maneri 1970] for the analysis of bubble motion in narrow inclined channels.

One of the major parameters measured by Maneri [1970] was the bubble rise velocity for different bubble volumes and corresponding to gradually increasing inclination angles, starting at $\theta=0^{\circ}$ for the vertical orientation of the conduit. A very interesting observation from the experiments was that for a range of different bubble sizes, the highest rise velocity always occurred at about $\theta \approx 45^{\circ}$.

Figure 10 shows the photos of bubbles for each of the above-mentioned situations.

Since the main objective of this study has been to quantify the effect of conduit inclination angle, the simulations have been performed for fixed: size conduit $(13.4 \mathrm{~mm}$ by $153 \mathrm{~mm}$ by $864 \mathrm{~mm})$ and bubble volume $\left(8 \mathrm{~cm}^{3}\right)$.

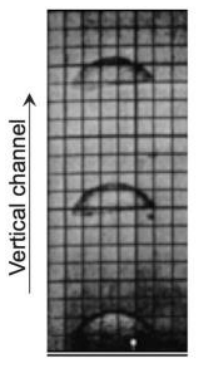

(a)

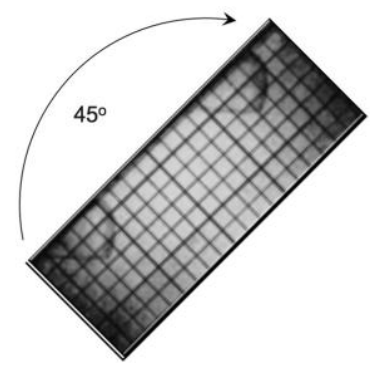

(b)
Figure 10. Sample photos of bubbles rising in narrow channels" (a) near vertical, (b) at $45^{\circ}$ inclination angle [Maneri 1970]. 
The computational domain, mesh and the initial bubble shape are shown in Figure 11 and Figure 12 for vertical and inclined channels. As can be seen, the same geometry was used for both situations.
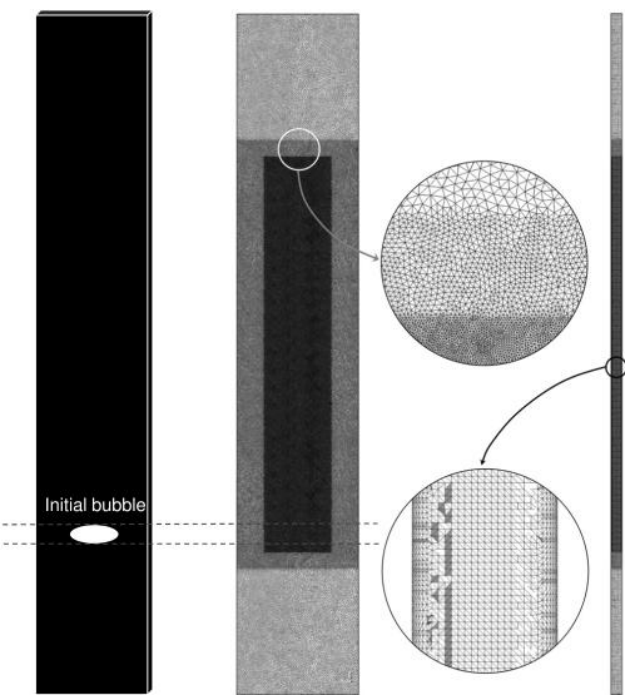

Figure 11. Computational domain, mesh and initial bubble shape for flow along a vertical channel.

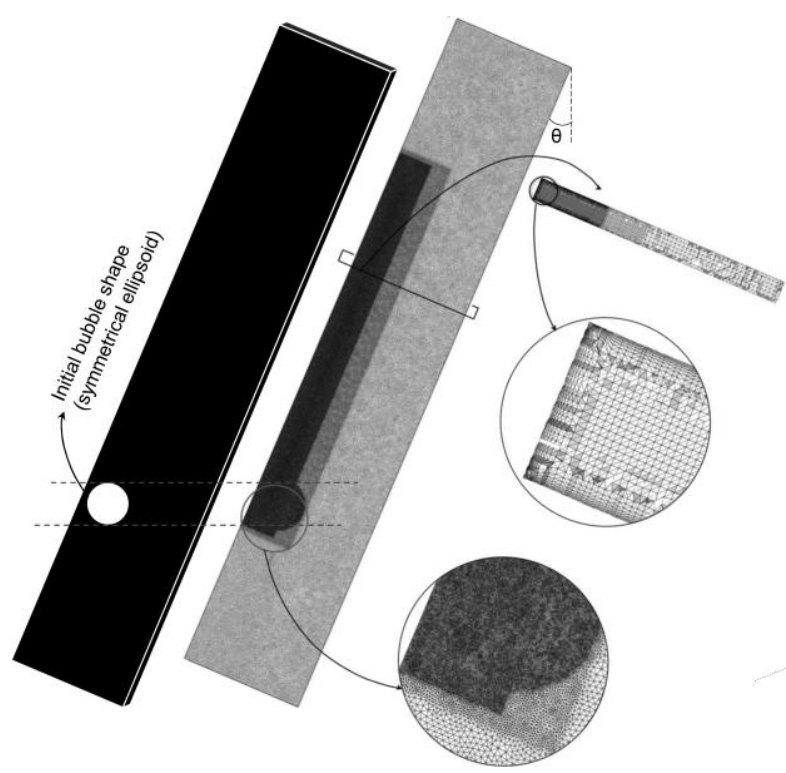

Figure 12. Computational domain, mesh and initial bubble shape for flow along an inclined channel.

The various prescribed values of the inclination angle were consistent with the reported experimental conditions. Table 2 shows the main parameters chosen to reproduce the experimental setup.

A symmetric ellipsoid and an elongated ellipsoid were used as the initial conditions for the bubble shape in the vertical and inclined cases; respectively. The initial bubble location was defined in such a way that the bubble did not touch any of the solid walls.

Table 2. Computational setup for bubbles rising in inclined narrow channels.

\begin{tabular}{lll}
\hline Channel Length & $\mathrm{cm}$ & 86.4 \\
Channel width & $\mathrm{cm}$ & 15.3 \\
Channel depth & $\mathrm{cm}$ & 1.34 \\
Temperature & ${ }^{\circ} \mathrm{C}$ & 20 \\
Liquid/Gas & & Water/Air \\
\hline
\end{tabular}

Different mesh designs have been used for the vertical and inclined orientations of the rectangular channel, to make sure that the bubble path is always sufficiently refined. The boundary layer mesh was applied on all solid walls including 7 layers with the first layer size and total length of $0.1 \mathrm{~mm}$ and 1.75 $\mathrm{mm}$; respectively.

Physically, the theoretical model represents a complete enclosure, so that the computational domain had no inlet or outlet. The bubble and liquid motion is due to the effect of buoyancy, combined with the shear force caused by the no-slip boundary condition at all walls. In the simulations, the initial velocity was set to zero and the system pressure was $100 \mathrm{kPa}$.

To facilitate and accelerate numerical convergence to the physical steady-state solution, the simulation was started with two phases having the same properties as water, and then the gas density and viscosity, as well as the surface tension, were gradually changed to their actual physical values during a short period of time. Multiple simulations have been performed for different inclination angles, starting with the vertical case.

Figure 13 shows a comparison between the bubble shape observed in the experiments and the one predicted by the present model.
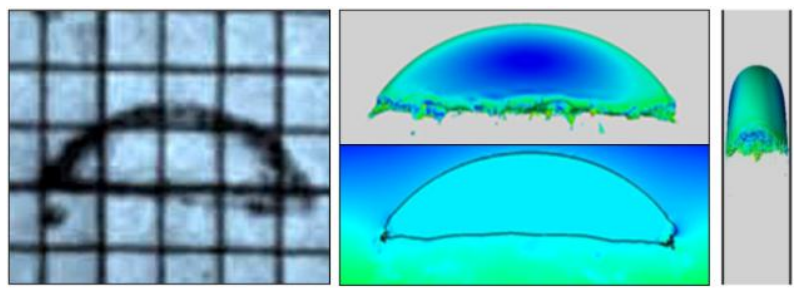

Figure 13. Bubble rising in a narrow near-vertical channel based on numerical simulation.

Figure 14 shows the simulation results for the channel inclined at $60^{\circ}$. For this and other inclination angles, the bubble shapes predicted by numerical simulations turned out to be quite similar to those observed in experiments performed by Maneri [1970].

Table 3 presents the results of numerical simulations compared to those of the measurements for the same bubble sizes of $8 \mathrm{~cm}^{3}$. 


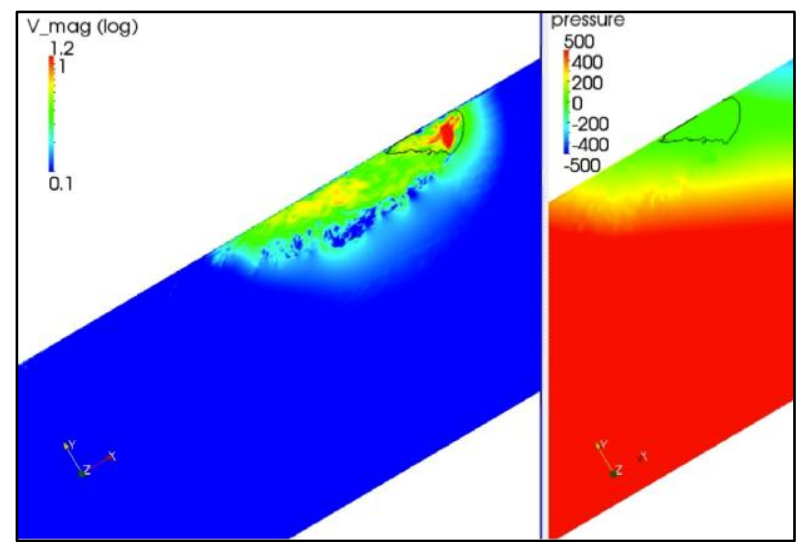

Figure 14. Bubble rising in a narrow channel inclined at $60^{\circ}$. The cross section of the domain is colored by velocity (logarithmic scale, left) and the pressure field (right). The interface is shown as a black line

Table 3. Bubble rise velocities in a narrow channel based on the experiments of Maneri [1970] and the PHASTA simulations for four inclination angles.

\begin{tabular}{llllcc}
\hline Inclination angle & $\mathbf{0}$ & $\mathbf{0}$ & $\mathbf{3 0}$ & $\mathbf{4 5}$ & $\mathbf{6 0}$ \\
\hline Experiment & $\mathbf{c m} / \mathbf{s}$ & 30.6 & 48.7 & 51.2 & 48.5 \\
Simulation & $\mathbf{c m} / \mathbf{s}$ & 30 & 42.1 & 43.8 & 41.3 \\
Difference & $\mathbf{\%}$ & 2.1 & 13.6 & 14.3 & 14.9 \\
\hline
\end{tabular}

As can be seen in table 3, even though the numerical results show the same trends as the experimental data, the predictions for inclined channels are not as accurate as those for the vertical case. It can be observed that as the inclination angle gets bigger, the numerical error increases and gets as high as $14.9 \%$ for the angle of $60^{\circ}$.

\subsection{Introduction of the Liquid Film}

A thorough analysis of the simulation results discussed in Section 5.1, including an inspection of the gas/liquid interface in the near-wall region, revealed that the liquid film between the bubble and the inclined wall above it was partially disappearing for all three inclined orientations of the channel. This in turn, slowed down the bubble motion.

A consistent physical explanation of the fact that accounting for the presence of the liquid film, rather that the film thickness, is a key factor for the proper model formulation. Such an explanation, based on both experimental observations and theoretical studies, is summarized below. Specifically, if a direct contact occurs between the gas and the solid wall, the net effect of the triple-line-induced force, which can be readily noticed by examining the difference between the advancing and receding contact angles, slows down the motion of both buoyancy-driven bubbles in inclined conduits [Podowski and Hirsa, 2001] and droplets sliding along inclined surfaces [Pierce et al., 2007]. In the latter case, the magnitude of such a force, which keeps droplets in place until the inclination angle exceeds a critical value, has been evaluated analytically [Vafaei and Podowski, 2004, 2005]. A similar analysis has been recently performed for gas bubbles under inclined walls [Jiao and Podowski, 2015], thus confirming the overall concept.

The formation of a triple line for a bubble in direct contact with the conduit wall is clearly seen in Figure 15(a), whereas Figure 15(b) shows a similar bubble but separated from the wall by a thin liquid film. For the same bubble volume and conduit inclination angle, the bubble velocity in the direct-contact case was about $20 \%$ lower than that for the liquid film case. For details, also see [Podowski, 2008]. The parameters characterizing thin liquid films have been investigated before, both experimentally [Podowski \& Hirsa, 2001] and theoretically [Podowski \& Kumbaro, 2004], and it has been found that the film thickness is of the order of 50 to $100 \mu \mathrm{m}$.

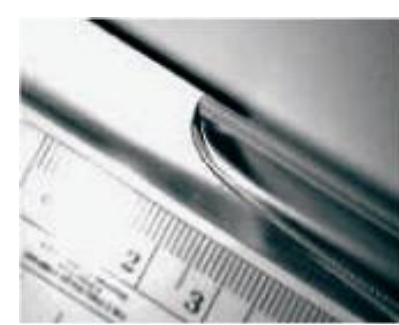

(a)

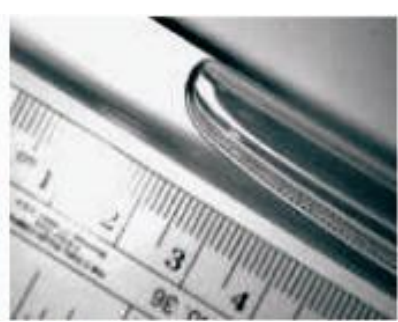

(b)
Figure 15. The shape of gas bubbles for two gas/liquid/solid conditions (a) nonwetting wall, (b) fully wetted wall [Podowski and Hirsa, 2001].

Overall, one concludes that the motion of large deformed bubbles along inclined walls mainly depends on the conduit wall wettability. If the wall is fully wetted, and the size of the conduit is large compared to the bubble size (so that the effective drag force is controlled by bubble shape and size rather than by wall shear) the liquid film is normally very thin and its precise thickness has a negligible effect on bubble motion. Since in the results shown in Figure 14, the disappearance of the liquid film above the bubble was numerically- rather than physically-driven, and taking into account that the liquid film thickness is below the resolution of even such a detailed grid as the one shown in Figure 12, an approach based on introducing a lubrication force has been used next to assure the presence of the liquid film. The method is based on adding an external repellent body force applied to a thin layer close to channel walls [Bolotnov et al, 2011]. Specifically, a force normal to the wall has been introduced for all inclination angles, with no specific 
fine-tuning or adjustments for each case, given by

$\vec{g}_{\text {repellent }}=-C \vec{g} \vec{n}$

where $\vec{n}$ is the vector normal to the solid surface toward the fluid. The coefficient, $\mathrm{C}$, smoothly changes from 100 on the solid surface to zero at $2 \mathrm{~mm}$ away from the wall. This force is only applied inside the blending region and not inside the pure liquid or gas phases.

The only criterion to define the magnitude of the body force was to make sure that the interface never touches the solid surfaces. Since, as mentioned above, the actual thickness of this super-thin film in inclined conduits has a negligible effect on the bubble shape and motion, the proposed modification, while being numerically consistent with the level-set formulation of the present model (which does not account for the liquid/solid and gas/solid molecular-level forces), is also consistent with the underlying micro-scale physics.

The results of PHASTA simulations using the updated model are shown in Figure 16 and Figure 17. The bubble shape, velocity field and pressure field are presented for the vertical channel and for the $30^{\circ}, 45^{\circ}$ and $60^{\circ}$ inclined channels.

It turns out that the bubble shape, as well as pressure and velocity fields for the vertical flow case are practically the same as those obtained without the lubrication force. On the other hand, as can be noticed by comparing Figure 14 and Figure 16(d), whereas for inclined channel orientations the macro-scale bubble shape is practically the same in both cases, the predicted bubble velocities are quite different. Specifically, when the liquid film practically disappears over a significant section of the bubble length, the predicted bubble velocity at the $60^{\circ}$ inclination angle, shown in Figure 14, is $41.3 \mathrm{~cm} / \mathrm{s}$, whereas if the film is maintained due to the effect of the external lubrication force (see Figure 16(d)), the corresponding velocity is $47.3 \mathrm{~cm} / \mathrm{s}$, which compares well against the measured velocity of $48.5 \mathrm{~cm} / \mathrm{s}$.

The lubrication effect of the liquid film is directly seen by comparing the bubble velocities for various inclination angles in Figure 17. As can be seen, the predicted bubble rise velocities are in a very good agreement with the measurements.

A detailed quantitative comparison is shown in Table 4 . The fact that the errors are lower than $2.5 \%$ is a clear indication that the new proposed model is capable of capturing the fluid mechanics of bubbles rising along inclined walls over a wide range of wall inclination angles.
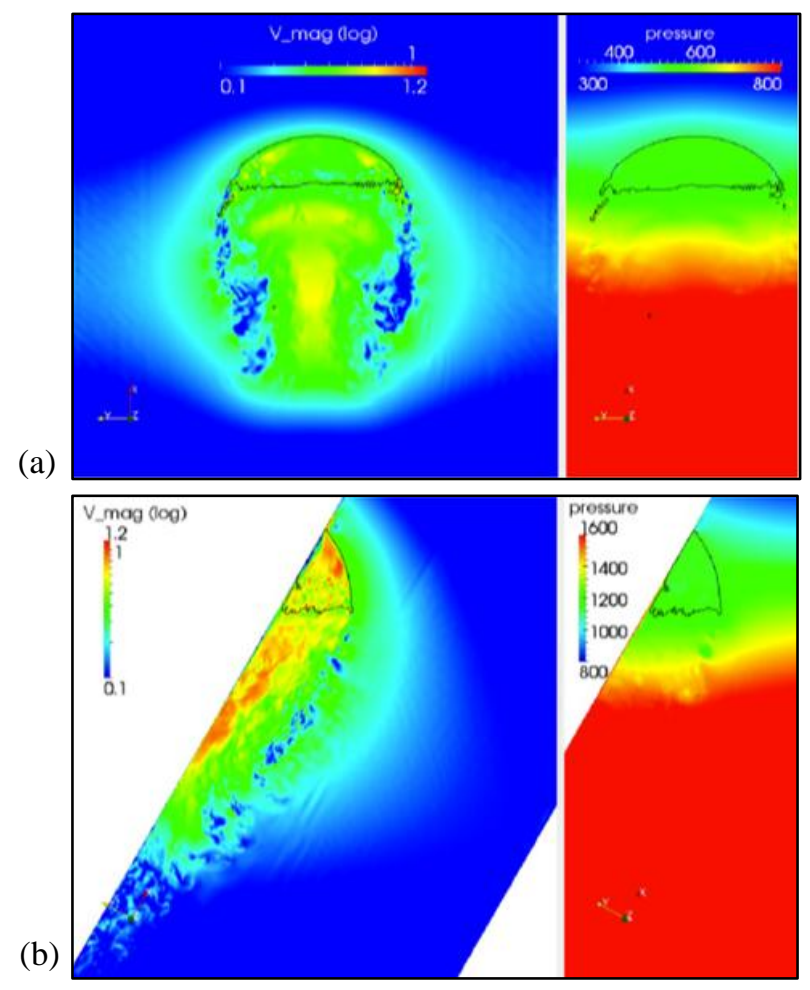

(b)

(c)

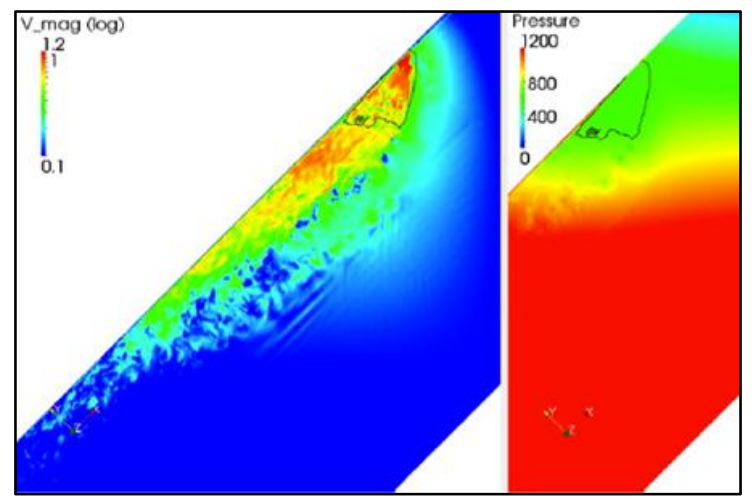

(d)

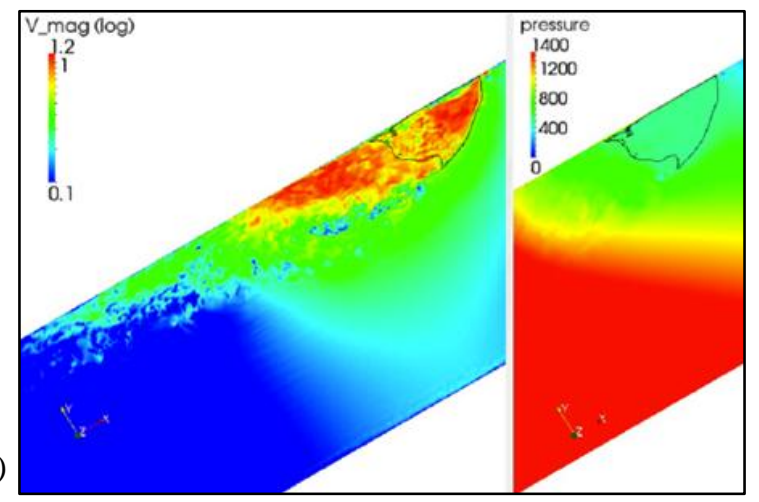

Figure 16. Bubbles rising in a narrow vertical channel and channels inclined at: (a) $0^{\circ}$ (b) $30^{\circ}$, (c) $45^{\circ}$, and (d) $60^{\circ}$ after introducing the liquid film. The domain cross section is colored by velocity (left, logarithmic scale) and the pressure field (right) with the interface as a black line. 


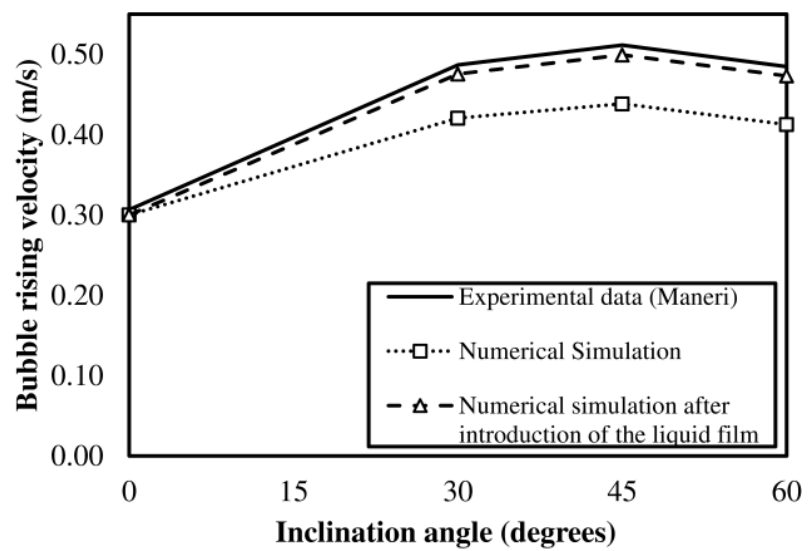

Figure 17. Bubble rise velocities in narrow channels based on the experiments of Maneri et al [1974] and on the numerical simulations before and after introducing the wall lubrication force.

Table 4. A comparison between the predicted and measured [Maneri et al 1974] bubble rise velocities in narrow channels for various inclination angles.

\begin{tabular}{llllll}
\hline Inclination angle & $\mathbf{0}$ & $\mathbf{0}$ & $\mathbf{3 0}$ & $\mathbf{4 5}$ & $\mathbf{6 0}$ \\
\hline Experimental & $\mathbf{c m} / \mathbf{s}$ & 30.6 & 48.7 & 51.2 & 48.5 \\
Numerical & $\mathbf{c m} / \mathbf{s}$ & 30.0 & 47.6 & 49.9 & 47.3 \\
Difference & $\mathbf{\%}$ & 2.1 & 2.3 & 2.4 & 2.4 \\
\hline
\end{tabular}

It is interesting to notice that the results shown in Figure 15 can also be used to explain the fact that the bubble rise velocity in vertical channels is lower than that in inclined channels. Namely, in the former case the numerical simulations show that two wakes are formed behind the bubble corners, but there is only one wake when the bubble moves along an inclined solid surface. This in turn, is an indication of a lower flow resistance (or drag) in inclined conduits, the effect of which exceeds the buoyancy force reduction over a considerable range of inclination angles. It can be concluded that if a proper liquid film is introduced between the bubbles and the solid walls, the numerical simulation of bubbles rising in vertical and inclined narrow channels is both physically consistent and in good quantitative agreement with the experimental observations.

\section{SUMMARY AND CONCLUSION}

A combined computational and analytical study has been performed on the multidimensional modeling of the motion of large deformable bubbles in conduits of various geometries and inclination angles.

Since even detailed computational models of twophase flow can no longer be called 'direct numerical methods' (due to the various constrains and assumptions introduced by the commonly used interface tracking methodologies), it is important that the results of numerical predictions be carefully checked for their physical consistency and limitations, rather than just compared against specific experimental results.

The focus of the present work was on the phenomena governing the dynamics of large bubbles moving in a quiescent liquid for two different conduit geometries: a vertical cylindrical pipe and a narrow rectangular channel of different orientations (inclination angles).

The computations have been performed using a combined DNS/Level-Set model implemented in the PHASTA code. The results of PHASTA simulations have been verified against simplified theoretical models and validated against experimental data. It has been demonstrated that the proposed approach is capable of accurately predicting the underlying fluid mechanics phenomena in a physically consistent and numerically efficient manner.

Given the complicated geometrical and operating conditions (a rectangular inclined channel, narrow in one direction but quite wide in the other), the present study utilized very large computational meshings to produce accurate results, and thus required the use of a supercomputer. However, it is interesting to mention that, it has already been shown that less detailed methods may be capable of producing quite reasonable (although likely less accurate) results for less demanding problems. Two such situations include vertical conduits [Anglart and Podowski, 2002(b)] and slightly inclined channels [Wierzbicki et al., 2007]. An issue which must be carefully addresses by any computational model deals with the effect of gas/liquid/solid properties on wall wettability and the presence of liquid film.

\section{ACKNOWLEDGMENT}

The authors would like to acknowledge the financial support provided to this study by the U.S. Department of Energy, and the computation resources provided by the Computational Center for Nanotechnology Innovations (CCNI) at Rensselaer Polytechnic Institute.

\section{REFERENCES}

Adalsteinsson, D. and Sethian, J. A., "The fast construction of extension velocities in level set methods," Journal of Computational Physics, Vol. 148, No. 1, pp. 2-22, 1999.

Anglart, H. and Podowski, M.Z., "Fluid Mechanics of Taylor Bubbles and Slug Flows in Vertical 
Channels", Nuclear Science \& Engineering, V.140, No.2, 2002(a), pp.165-173.

Anglart, H. and Podowski, M.Z., "On the Multidimensional Modeling of Gas-Liquid Slug Flows", Proc. Twelfth International Heat Transfer Conference, Grenoble, France, 2002(b).

Barnea, D., "Effect of Bubble Shape on Pressure Drop Calculations in Vertical Slug Flow", International Journal of Multiphase Flow, Vol. 16, No. 1, pp. 79-89, 1990.

Behafarid, F., Bolotnov, I. A., Podowski, M. Z. and Jansen, K. E., "Two phase cross jet in a fuel rod assembly using DNS/level-set method," in 7th International Conference of Multiphase Flow (ICMF), Tampa, FL, 2010.

Behafarid, F., Shaver, D., Bolotnov, I. A., Jansen, K. E., Antal, S. P. and Podowski, M. Z., "Multiscale approach to the modeling of fission gas discharge during hypothetical loss-of-flow accident in Gen-iv sodium fast reactor," International Congress on Advanced Nuclear Power Plants (ICAPP), Chicago, IL, 2012.

Behafarid, F., "Multi-scale multi-physics simulation of gas injection into the liquid using DNS/LSM," PhD dissertation. Mechanical Engineering Department, Rensselaer Polytechnic Institute of Technology, Aug. 2012.

Behafarid, F., Shaver, D., Bolotnov, I. A., Antal,S. P., Jansen, K. E. and Podowski, M. Z., "Coupled DNS/RANS Simulation of Fission Gas Discharge During Loss-of-Flow Accident in Generation IV Sodium Fast Reactor," Journal of Nuclear Technology, Vol. 181, No. 1, pp. 44-55, 2013.

Bendiksen, K., "On the motion of long bubbles in vertical tubes," International Journal of Multiphase Flow, Vol. 11, No. 6, pp. 797-812, 1985.

Bolotnov, I. A., Behafarid, F., Shaver, D., Guo, T., Wang, S., Wei, H., Antal,S. P., Jansen, K. E., Samulyak, R. and Podowski, M. Z., "Interaction of computational tools for multiscale multiphysics simulation of generation-IV reactors," International Congress on Advanced Nuclear Power Plants (ICAPP), San Diego, CA, 2010.

Bolotnov, I. A., Jansen, K. E., Drew, D. A., Oberai, A. A., Lahey, R. T. and Podowski, M. Z., "Detached direct numerical simulations of turbulent two-phase bubbly channel flow," International Journal of Multiphase Flow, Vol. 37, No. 6, pp. 647-659, 2011.

Bolotnov, I. A., Behafarid, F., Shaver, D., Antal,S. P., Jansen, K. E. and Podowski, M. Z., "Coupled DNS/RANS simulation of fission gas discharge during loss-of-flow accident in gen-IV sodium fast reactor,' 14th International Topical Meeting on Nuclear Reactor Thermal-hydraulics (NURETH-14), Toronto, Ontario, Canada, 2011.

Brackbill, J. U., Kothe, D. B. and Zemach, C., "A continuum method for modeling surface tension,"
Journal of Computational Physics, Vol. 100, Issue 2, pp. 335-354, 1992.

Dukler, A. E. and Bergelin, O. P., "Characteristics of flow in falling liquid films," Chemical Engineering Progress, Vol 48, pp 557-563, 1952.

Fabre, J. and Liné, A., "Modeling of two-phase slug flow," Annual Review of Fluid Mechanics, Vol. 24, pp 21-46, 1992.

Fourar M., and Bories, S., "Experimental study of air-water two-phase flow through a fracture (narrow channel)," International Journal of Multiphase Flow, Vol. 21, No. 4, pp. 621-637,1995.

Franca, L. P. and Frey, S. L., "Stabilized FiniteElement Methods. II. The Incompressible NavierStokes Equations," Computer Methods in Applied Mechanics and Engineering, Vol. 99, Issue 2-3, pp. 209-233, 1992.

Hibiki, T., Situ, R., Mi, Y. and Ishii, M., "Local flow measurements of vertical upward bubbly flow in an annulus," International Journal of Heat and Mass Transfer, Vol. 46, No. 8, pp. 1479-1496, 2003.

Jansen, K. E., Collis, S. S., Whiting, C. and Shakib, F., "A better consistency for low-order stabilized finite element methods," Journal of Computer Methods in Applied Mechanics and Engineering, Vol. 174, pp. 153-170, 1999.

Jansen, K. E., Whiting, C. H. and Hulbert, G. M., "A generalized-alpha method for integrating the filtered Navier-Stokes equations with a stabilized finite element method," Computer Methods in Applied Mechanics and Engineering, Vol. 190, No. 3-4, pp. 305-319, 2000.

Jiao, H. and Podowski, M.Z., "Theoretical analysis on the effect of gas/liquid/solid interfacial forces on bubble shape in inclined conduits," in preparation for submission to Physics of Fluids, 2015.

Karapantsios, T. D., and Karabelas, A. J., "Longitudinal characteristics of wavy falling films," International Journal of Multiphase Flow, Vol 21(1), pp 119-127., 1995

Llewellin, E. W., Del Bello, E., Taddeucci, J., Scarlato, P., \& Lane, S. J., "The thickness of the falling film of liquid around a Taylor bubble," Proceedings of the Royal Society A: Mathematical, Physical and Engineering Sciences, 468 (2140), 2011.

Maneri, C. C., "The motion of plane bubbles in inclined ducts," Ph.D. dissertation, Mechanical Engineering Department, Polytechnic Institute of Brooklyn, 1970.

Maneri, C. C. and Zuber, N., "An experimental study of plane bubbles rising at inclination," International Journal of Multiphase Flow, pp. 623-645, 1974.

Nagrath, S., "Adaptive stabilized finite element analysis of multi-phase flows using level set approach," PhD dissertation, Mechanical Engineering Department, Rensselaer Polytechnic Institute, 2004. 
Nagrath, S., Jansen, K. E. and Lahey, R. T., "Computation of incompressible bubble dynamics with a stabilized finite element level set method," Computer Methods in Applied Mechanics and Engineering, Vol. 194, No. 42-44, pp. 4565-4587, 2005.

Pierce, E., Carmona, F.J., and Amirfazli, A., "Understanding of and contact angle results in tilted plate experiments," Colloids and Surfaces, 323, pp. 7382, 2008.

Polonsky, S., Shemer L., Barnea, D.,'The relation between the Taylor bubble motion and the velocity field ahead of it," International Journal of Multiphase Flow, Vol 25, pp 957-975, 1999.

Podowski M. Z. and Hirsa, A., "The Effect of Nanoparticles on Taylor-Bubble Motion in Inclined Pipes," Proc. 4th Int. Conf. on Multiphase Flow, ICMF'2001, New Orleans. LA., 2001.

Podowski M. Z. and Kumbaro, A., "The modeling of thin liquid films along inclined surfaces," Journal of Fluids Engineering, Vol. 126, pp. 565-572, 2004.

Podowski, M.Z., "Multidimensional Modeling of Two-Phase Flow and Heat Transfer," International Journal of Numerical Methods for Heat \& Fluid Flow, V. 18, Issue 3/4, 2008, pp.491-513.

Rodriguez J. M., "Numerical simulation of twophase annular flow," $\mathrm{PhD}$ dissertation, Mechanical Engineering Department, Rensselaer Polytechnic Institute, 2009.

Rodriguez, J. M., Sahni, O., Lahey, R. T. and Jansen, K. E. "A parallel adaptive mesh method for the numerical simulation of multiphase flows," Computers and Fluids, Vol. 87, pp. 115-131, 2013.

Sahni, O., Mueller, J., Jansen, K. E, Shephard, M. S. an Taylor, C. A., "Efficient anisotropic adaptive discretization of the cardiovascular system," Computer Methods in Applied Mechanics and Engineering, Vol. 195, No. 41-43, pp. 5634-5655, 2006.

Sethian, J. A., "Level Set Methods and Fast Marching Methods," Cambridge University Press, 1999.

Sussman, M., Fatemi, E., Smereka, P. and Osher, S., "An improved level set method for incompressible two-phase flows," Journal of Computers and Fluids, Vol. 27, Issue 5-6, pp. 663-680, 1998.

Sussman, M. and Fatemi, E., "An efficient, interface-preserving level set re-distancing algorithm and its application to interfacial incompressible fluid flow," Siam Journal on Scientific Computing, Vol. 20, Issue 4, pp. 1165-1191, 1999.

Sussman, M., Almgren, A. S., Bell, J. B., Colella, P., Howell, L. H. and Welcome., M. L., "An adaptive level set approach for incompressible two-phase flows," Journal of Computational Physics, Vol. 148, Issue 1, pp. 81-124, 1999.

Taylor, C. A., Hughes, T. J. R. and Zarins, C. K., "Finite element modeling of blood flow in arteries," Computer Methods in Applied Mechanics and
Engineering, Vol. 158, Issues 1-2, pp. 155-196, 1998.

Tejada-Martinez A. E. and Jansen, K. E., "A parameter free dynamic subgrid-scale model for largeeddy simulation," Computer Methods in Applied Mechanics and Engineering, Vol. 194, No. 9, pp. 12251248, 2005.

Vafaei, S. and Podowski, M.Z., "The Modeling of Liquid Droplet Shape on Horizontal and Inclined Surfaces," Proc. 5th International Conference on Multiphase Flow, ICMF'04, Yokohama, Japan, MayJune, 2004.

Vafaei, S. and Podowski, M.Z., "Analysis of the Relationship between Liquid Droplet Size and Contact Angle," Advances in Colloid and Interface Science, 113, pp.133-146, 2005.

Whiting, C. H., "Stabilized finite element methods for fluid dynamics using a hierarchical basis," $\mathrm{PhD}$ dissertation, Mechanical Engineering Department, Rensselaer Polytechnic Institute, 1999.

Whiting C. H. and Jansen K. E., "A stabilized finite element formulation for the incompressible NavierStokes equations using a hierarchical basis," Journal of Numerical Methods in Fluids, pp. 93-116, 2001.

Wierzbicki, B.W., Antal, S.P. and Podowski, M.Z., "On the Modeling of Gas Bubble Evolution and Transport using Coupled Level-Set/CFD Method," Nuclear Technology, 158, 2, 2007, pp.261-274.

Wilmarth T. and Ishii, M., "Two-phase flow regimes in narrow rectangular vertical and horizontal channels," International Journal of Heat and Mass Transfer, Vol. 37, No. 12, pp. 1749-1758, 1994.

Yadigaroglu, G., Simiano, M., Milenkovic, R. V., Kubasch, J., Milelli, M., Zboray, R., De Cachard, F., Smith, B., Lakehal, D. and Sigg, B., "CFD4NRS with a focus on experimental and CMFD investigations of bubbly flows," Nuclear Engineering and Design., Vol. 238, No. 3, pp. 771-785, 2008.

Zukoski, E., "Influence of viscosity, surface tension, and inclination angle on motion of long bubbles in closed tubes," Journal of Fluid Mechanics, Vol. 25, Issue 4, pp. 821-837, 1966. 